\title{
Persistence of Diarrheal Pathogens Is Associated with Continued Recruitment of Plasmablasts in the Circulation
}

\author{
Anu Kantele $e^{1,2,3}$ \\ ${ }^{1}$ Division of Infectious Diseases, Department of Medicine, Helsinki University Central Hospital, Aurora Hospital, \\ Building 5, 3rd floor, POB 348, 00029 Helsinki, Finland \\ ${ }^{2}$ Department of Bacteriology and Immunology, Haartman Institute, University of Helsinki, 00014 Helsinki, Finland \\ ${ }^{3}$ Institute of Clinical Medicine, University of Helsinki, 00014 Helsinki, Finland \\ Correspondence should be addressed to Anu Kantele, anu.kantele@hus.fi
}

Received 1 July 2011; Revised 27 September 2011; Accepted 8 October 2011

Academic Editor: Daniel Mucida

Copyright () 2012 Anu Kantele. This is an open access article distributed under the Creative Commons Attribution License, which permits unrestricted use, distribution, and reproduction in any medium, provided the original work is properly cited.

\begin{abstract}
Intestinal antigen encounter leads to recirculation of antigen-specific plasmablasts via lymphatics and blood back to the intestine. Investigating these gut-originating cells in blood provides a less invasive tool for studying intestinal immune responses, with the limitation that the cells disappear from the circulation in two weeks. No data exist on situations where pathogens persist in the intestine. Patients with Salmonella, Yersinia, or Campylobacter gastroenteritis and volunteers receiving an oral typhoid vaccine were assayed for plasmablasts specific to each subject's own pathogen/antigen weekly until the response faded. In vaccinees, plasmablasts disappeared in two weeks. In gastroenteritis, the response faded 2-3 and 3-7 weeks after the last positive Salmonella or Yersinia stool culture. Even in symptomless patients, pathogens persisting in the intestine keep seeding plasmablasts into the circulation. Assaying these cells might offer a powerful tool for research into diseases in which persisting microbes have a potential pathogenetic significance.
\end{abstract}

\section{Introduction}

The intestine represents the largest immunological tissue in the body and carries the majority of all lymphocytes $[1,2]$. Pathogens encountered in the intestine activate antigen-specific lymphocytes in Peyer's patches, and these cells migrate to mesenteric lymph nodes and further via lymphatics and blood to the intestinal lamina propria as effector lymphocytes [2-6]. Consistent with this recirculation of activated intestinal lymphocytes, antigen-specific effector lymphocytes have been found in the circulation after intestinal antigen encounter both after oral [7-11] and rectal $[10,12]$ vaccinations and in intestinal infections [13-15].

The mechanisms underlying this recirculation of activated intestinal lymphocytes have been a subject of extensive research. It has been shown that dendritic cells in the intestine present the antigens to lymphocytes in Peyer's patches and program these cells to express a set of receptors determining their later migratory behavior [2-6]. Next, these activated lymphocytes migrate to mesenteric lymph nodes and return to the mucosal sites with the help of lymphatics and blood. This return once appeared to occur randomly with circulating blood, but, in fact, it exhibits marked tissue selectivity at the final stage of homing from blood through the endothelium into the tissues. This homing is a multistep process requiring an interaction of lymphocyte surface molecules recognizing their ligands distributed in a tissue-specific manner in the body [2-6]. Lymphocytes homing to the intestine express both CCR9 $[1-3,16]$, a chemokine receptor mediating homing to the small intestine, and $\alpha_{4} \beta_{7}[1-3,17]$, a gut-specific homing receptor (HR) that recognizes Mad-CAM1 on the endothelial venules of the intestine. The majority of gut-originating antigenspecific plasmablasts found in the peripheral blood after oral vaccination [9-11] or in intestinal infections [14] have been shown to express the gut $\mathrm{HR}, \alpha_{4} \beta_{7}$, implying a preferential homing of these cells to the intestinal lamina propria.

Research into intestinal immune responses in the human gut has been hampered by the instability of antibodies in secretions and, to an even greater extent, by ethical 
restrictions. Investigation of gut-originating plasmablasts in the peripheral blood circumvents some of these problems. Plasmablasts (preplasma cells) are close to end-stage cells of the B-cell lineage representing only a minor part of all circulating B cells: recently activated and developed into effector cells, they constitute the part that actively secretes antibodies while being on their way to settling down in the target tissues as plasma cells. Plasmablasts recently activated in the intestine are identified in the circulation as antibody-secreting cells (ASCs) with a spontaneous secretion of antibodies against intestinally encountered antigens [715]. Studies with oral vaccines have shown that these cells can be caught from the peripheral blood 3-5 days after the original antigen encounter, they peak in number around day 7 and disappear within two weeks $[7,8,18]$, consistent with their homing back to the mucosa. Catching gut-originating effector lymphocytes from the circulation has proved a useful tool in studying mucosal immune responses to both oral vaccines [7-11] and intestinal infections [13-15]. However, a major restriction to this approach is the transient nature of the response: the actual window for catching the cells is no longer than a few days. The kinetics has been explored with oral vaccinations $[7,8,18]$ where the exact time of antigen encounter is known and the vaccine antigen persists in the intestine for only a short time. The present study investigates the response in natural infections where the pathogen persists in the intestinal milieu for a longer period of time. This is of interest also in view of diseases in which pathogen persistence plays a role in the pathogenesis of the disease.

\section{Methods}

Patients with diarrhea and volunteers receiving an oral typhoid vaccine were studied for circulating plasmablasts specific to the pathogen isolated from their own stool sample or to the vaccine antigen, respectively. The kinetics of the response was studied along with determinations of isotypes. In order to confirm the intestinal homing commitment of these migrating cells, the homing profile was determined in a subgroup of volunteers in both groups.

2.1. Patients. 23 patients attending the Central Hospital of Central Finland or the Helsinki University Central Hospital and six healthy volunteers were enrolled in the study. Informed consent was obtained from each patient/volunteer before participation. The study was conducted according to the principles stated in the Helsinki Declaration on human experimentation, and the study protocol was reviewed and approved by the Ethics Committees of the participating hospitals.

22 patients ( 12 women, 10 men, aged $36-63$ years) had bacterial diarrhea, diagnosed on the basis of watery stools and a pathogen isolated from stool samples. One patient had no diarrhea but was examined because of her spouse's positive Salmonella culture: her stool culture proved positive for the same pathogen. In her case, the day of the first positive stool sample was defined as day 0 , while, for all others, day 0 indicates the day of the first symptoms. None of the patients had a known previous history of diarrhea caused by a bacterial pathogen, nor had they been diagnosed with immune deficiencies or other significant underlying diseases. Six healthy volunteers (all women, aged 24-47), who had not received the typhoid vaccination previously, were each given the oral Salmonella typhi Ty21a vaccine (Vivotif, Crucell, Switzerland) in three doses two days apart, according to the manufacturer's instructions. For vaccinees, the day of the first vaccine dose was marked as day 0 .

2.2. Collection of Blood Samples, Isolation of PBMC, and Preparation of Antigen. For patients with diarrhea, first blood samples for ASC analyses were drawn 6-33 days after the onset of the symptoms, and, for vaccinees, a series of blood samples was drawn $0,5,7,9,12,14$, and 16 days after the oral typhoid vaccination and then, in both groups, weekly for as long as the ASCs were detected or the pathogen could be recovered from the stool samples, and the patient was available. Mononuclear cells were isolated from heparinized blood by Ficoll-Paque density-gradient centrifugation as described previously [8] and adjusted to a concentration of $2 \times 10^{6}$ cells $/ \mathrm{mL}$. HR expressions were determined in the first blood sample drawn in five patients with diarrhea and on day seven after vaccination in five vaccinees.

Bacterial strains identified in the stool samples were grown on nutrient agar plates and formalin-killed as described previously $[13,14,19,20]$. The concentration of the bacterial suspension was adjusted to $10^{8}$ bacteria $/ \mathrm{mL}$.

2.3. Separation of Receptor-Negative and Receptor-Positive Cell Populations. The separation of the cells into receptornegative and receptor-positive populations has been described earlier $[9,14,21]$. Briefly, the cells were incubated with the monoclonal antibodies anti- $\alpha_{4} \beta_{7}$ (ACT-1) (Millennium Pharmaceuticals, Cambridge, MA), anti-L-selectin (Leu8) (Becton-Dickinson), or anti-CLA (HECA-452) (received from Dr. Sirpa Jalkanen, University of Turku, Finland), washed twice, and incubated with Dynal M-450 magnetic beads coated with sheep anti-mouse IgG (Dynal, Oslo). The beads with the attached cells were separated from the suspension by applying a magnet outside the test tubes and the supernatants with the receptornegative cells collected. The receptor-positive cells attached to the beads were collected. Both the receptor-positive and receptor-negative cell populations were immediately analyzed with the enzyme-linked immunospot (ELISPOT) assay for numbers of ASC.

The efficiency of the cell separations was checked in pilot experiments as described previously $[9,14]$.

2.4. Assay of Specific Antibody-Secreting Cells. The total population of PBMC and the receptor-positive and receptornegative cell populations were each assayed for specific ASC with ELISPOT: the assay of specific ASC has been described previously $[8,13,14,19,20]$. In brief, for patients with diarrhea, 96-well microtiter plates (Maxisorp, Nunc. 
Denmark) were coated with a whole-cell preparation of formalin-killed pathogen isolated from the stool sample of the same patient (see above) or, for vaccinees, with a preparation formalin-killed Salmonella strain SL2404 carrying the $\mathrm{O}$-antigen 9 and 12 similarly to the vaccine strain Ty21a. The cells were incubated in the wells, and antibodies secreted during this time detected with alkaline phosphataseconjugated anti-human IgA (Sigma-Aldrich, Mo, USA), IgG (Sigma-Aldrich), and IgM (SouthernBiotech, Birmingham, AL, USA) followed by application of substrate (bromo-4chloro-3-indolyl phosphate p-toluidine salt; Sigma-Aldrich) in melted agarose. Specific ASCs were enumerated by counting the spots in the wells in a light microscope. A response was defined as $>2$ specific $\mathrm{ASC} / 10^{6} \mathrm{PBMC}$ in at least one sample.

2.5. Statistics. The numbers of ASC were calculated as geometric means \pm SEM. The proportions of the receptorpositive ASC were calculated as follows: \% of receptorpositive cells among ASC $=(100 \mathrm{X}$ the number of ASC in receptor-positive population) $\div$ (the sum of the number of ASC in receptor-positive and receptor-negative populations). Statistical comparisons were carried out using Student's $t$ test. Results of statistical analyses were considered significant when $P<0.05$.

\section{Results}

3.1. Pathogens and Symptoms. In patients with gastroenteritis, Salmonella enteritidis was grown as a pathogen in stool culture in 10/23 cases, Yersinia enterocolitica in 7/23, Campylobacter jejuni in $2 / 23$, and S. stockholm, S. bradford, S. emek, and $S$. typhimurium each in one case. In 12/23 patients, only one positive stool sample was obtained, and the second one taken 1-2 weeks later was negative. In 11/23 patients, 211 positive stool samples were collected. $12 / 23$ patients had symptoms during the first sampling, and 10/23 of them until $1-5$ weeks after it. In $3 / 23$, patients the symptoms had faded away one week, and in $8 / 23$ patients in $2-4$ weeks before the first blood sample.

3.2. General Characteristics of the Antigen-Specific Plasmablast Response. All six volunteers vaccinated with Ty21a showed a vaccine-antigen-specific ASC response similar to that in our previous studies $[8,12]$ (Figure 1).

A response of circulating pathogen-specific plasmablasts was found in $8 / 10$ patients with Salmonella enteritidis (Figure 2). In one patient, there was no response despite a positive stool sample one week before (symptoms started 22 days earlier). In one patient, the blood sample was obtained only four weeks after the last positive stool sample and last symptoms. Circulating ASCs were detected in $5 / 7$ patients with Yersinia enterocolitica (Figure 3). In one patient with a clear response, no further follow-up samples could be obtained. No ASCs were found in 2/7 patients: in both of these cases, the sample was drawn one week after the symptoms had faded and four weeks after the last positive stool sample.

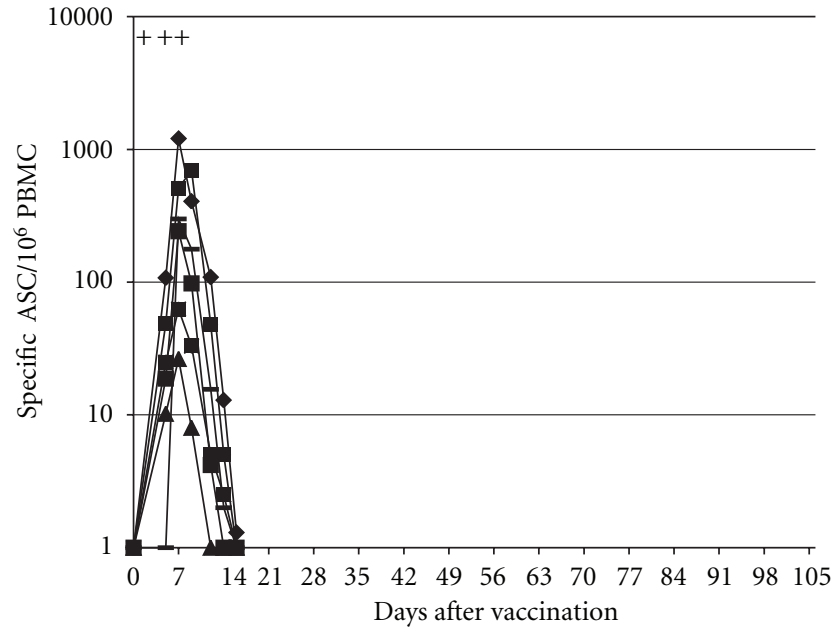

FIGURE 1: Numbers of vaccine antigen $(\mathrm{O} 9,12)$-specific circulating plasmablasts identified as antibody-secreting cells $/ 10^{6} \mathrm{PBMC}$ (IgA+ IgG- + IgM) in six volunteers vaccinated with the live oral Salmonella typhi Ty21a vaccine. Each volunteer received one vaccine dose on days 0,2 , and 4 (the vaccination days are indicated with “+”). To determine the kinetics of the response, the numbers of plasmablasts were determined on several days for as long as ASCs were found in the samples. The values of each individual are connected with a line.

Patients with S. bradford, S. emek, and S. typhimurium as pathogens had a response (Figures $4(\mathrm{a})-4(\mathrm{c})$ ). The patient with Salmonella stockholm did not show a response even though the samples were drawn one week after positive stool culture and last symptoms. One patient with Campylobacter jejuni had a vigorous response (10000 ASC/106 PBMC) (Figure $4(\mathrm{~d})$ ), while the other one showed no response; her blood sample was drawn three weeks after the last positive stool sample.

Among the vaccinees, IgA-ASC predominated in three volunteers, IgG in two, and IgM in one. The geometric mean of the peak of the responses (IgA + IgG + IgM-ASC) was $215 \pm 184 / 10^{6}$ PBMC. The isotype distribution is shown in Figure 5.

Out of all patients, the response was dominated by IgA in $15 / 17$ cases and IgM in 2/17 cases (one with S. typhimurium and one with Yersinia enterocolitica). The geometric mean of the peak of the responses (IgA + IgG + IgM-ASC) was $64 \pm$ $70 / 10^{6} \mathrm{PBMC}$ in patients with S. enteritidis and $459 \pm 614 / 10^{6}$ PBMC with Yersinia enterocolitica. The isotype distributions are shown in Figure 5.

3.3. Kinetics of the Antigen-Specific Plasmablast Response. In the vaccinees, the response peaked on day 7 and declined thereafter. No samples were drawn after day 16, since the response had faded away/was negligible already on that day in all volunteers (Figure 1).

In patients with gastroenteritis, the numbers of antigenspecific ASC were followed weekly for as long as the response persisted or the stool culture remained positive, and the patient was available. The highest number of ASC was in 


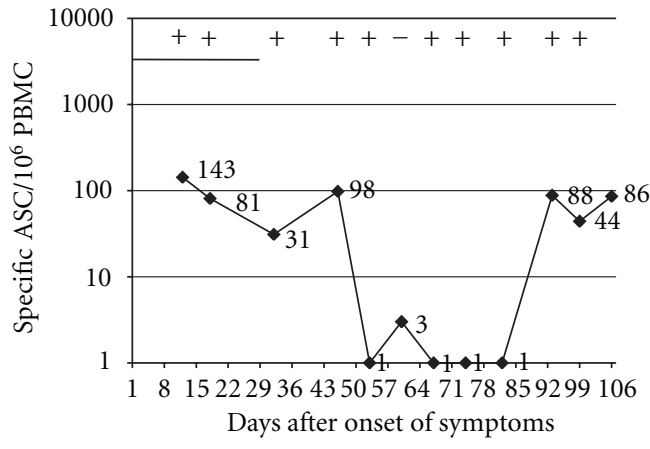

(a)

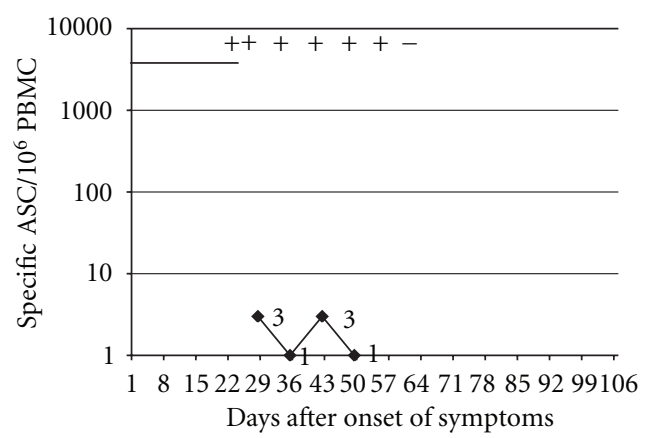

(c)

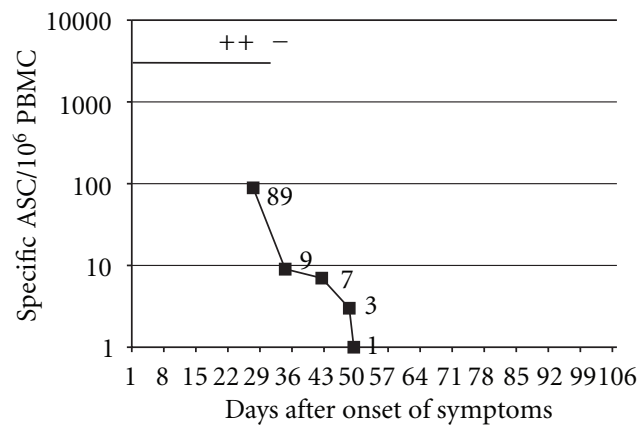

(e)

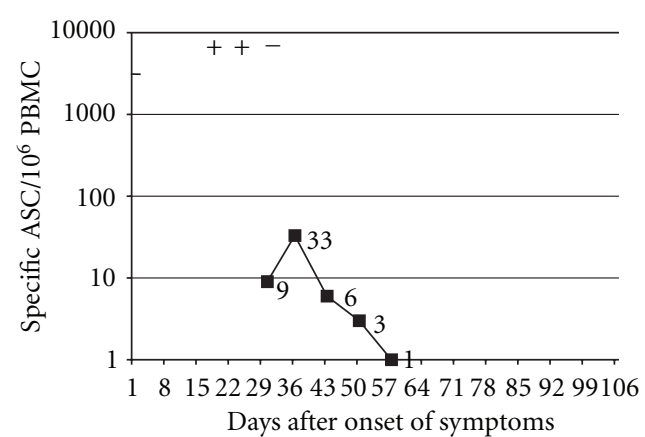

(g)

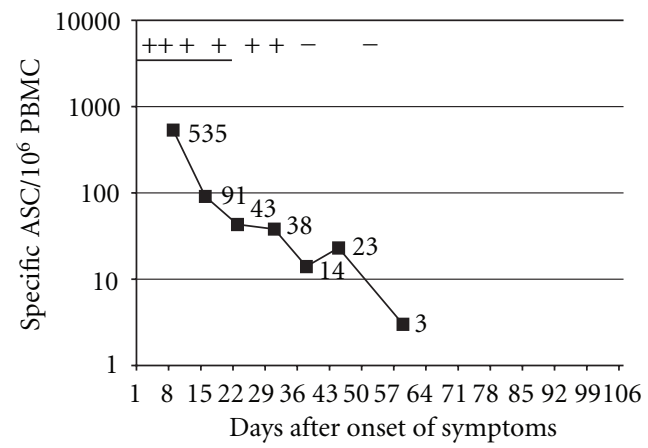

(b)

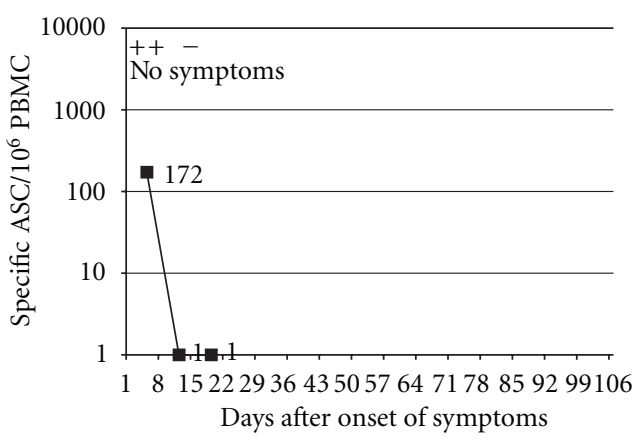

(d)

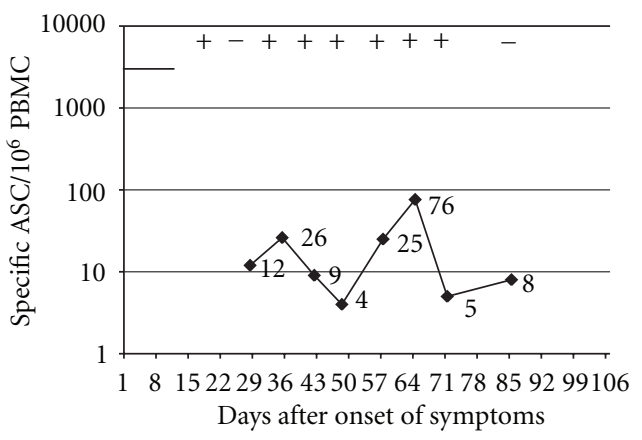

(f)

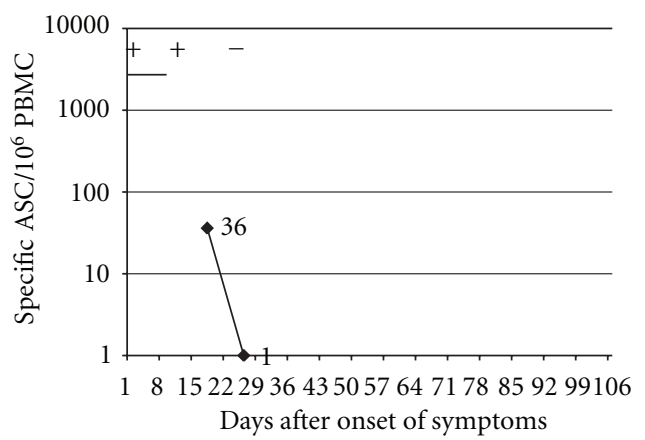

(h)

FIGURE 2: Relation of pathogen-specific circulating plasmablasts (black curve) with symptoms (black horizontal line) and findings in stool samples (+ or - ) in eight patients with gastroenteritis caused by Salmonella enteritidis. To determine the kinetics of the response, the numbers of plasmablasts were assessed on several occasions as long as they were found in the samples or the pathogen could be isolated from the stool samples, and the patient was available. The plots represent each data from one individual. The day of the onset of the symptoms was marked as day 0 . 


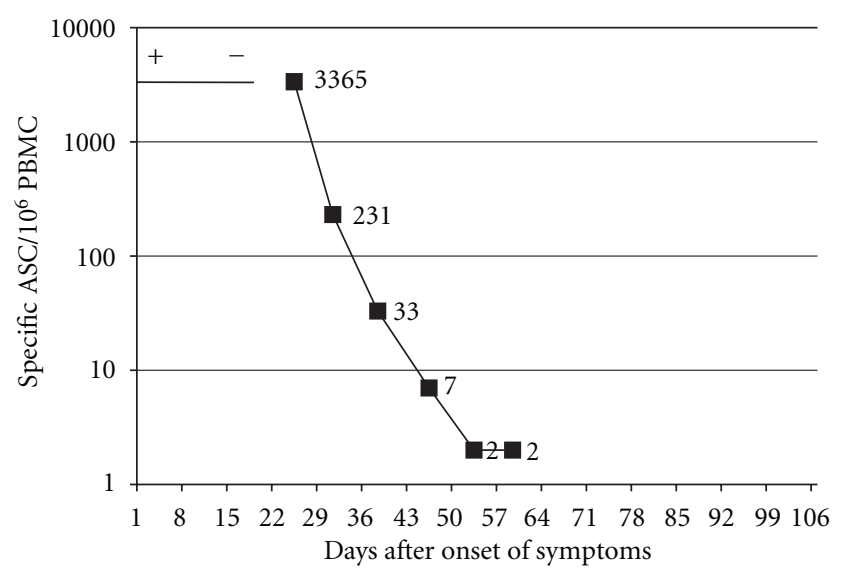

(a)

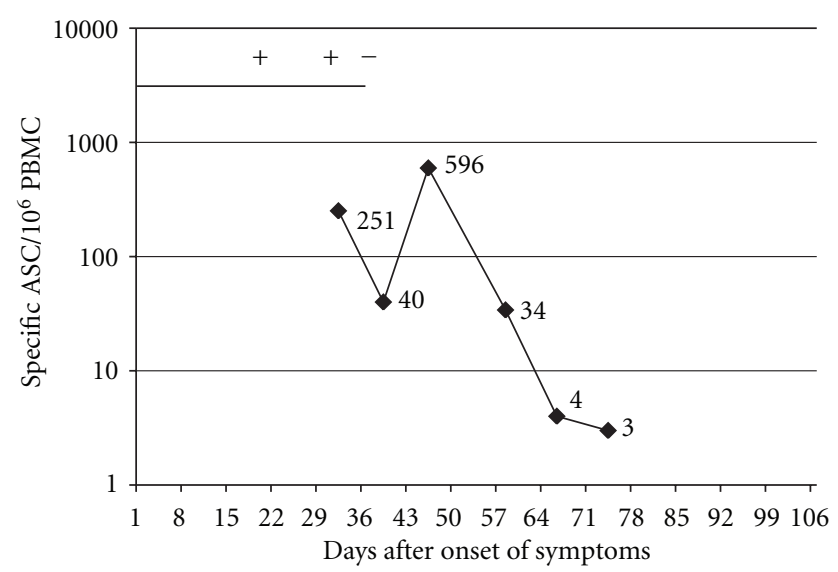

(c)

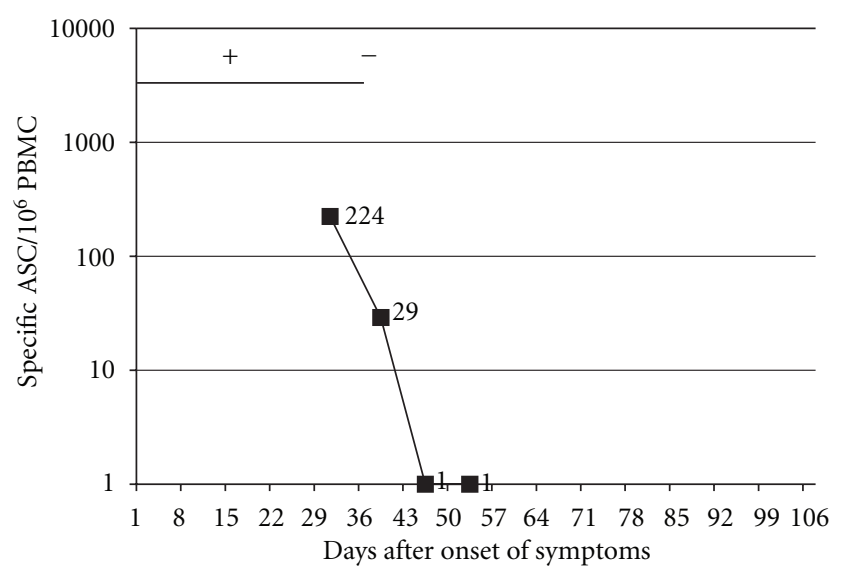

(b)

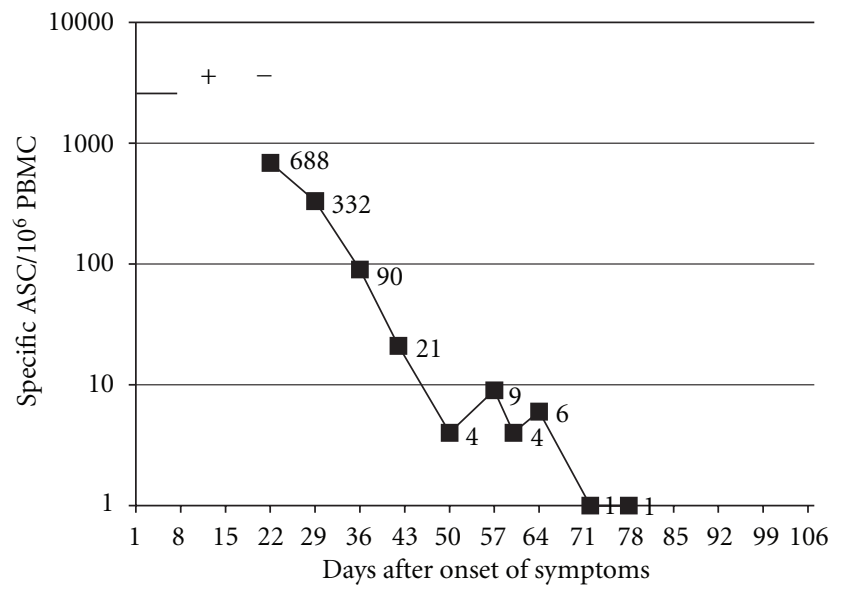

(d)

FIGURE 3: Relation of pathogen-specific circulating plasmablasts (black curve) with symptoms (black horizontal line) and findings in stool samples (+ or - ) in four patients with gastroenteritis caused by Yersinia enterocolitica. The plots represent each data from one individual. The day of the onset of the symptoms was marked as day 0 .

$12 / 16$ cases found in the first sample drawn, that is, soon after the beginning of symptoms and after that the magnitude of the response appeared to fluctuate until it faded away (Figures 2-4). The numbers of weekly blood samples drawn varied from one till up to 12 (Figures 2-4). The fading of ASC appeared to be associated with the stool samples turning negative, rather than the subsiding of the symptoms, as the ASC response was prolonged in some volunteers with persisting positive stool cultures even after the symptoms had subsided (Figures 2(a), 2(c), 2(f)). The response appeared to fade away faster after Salmonella (2-3 weeks) (Figure 2) than after Yersinia infection (3-7 weeks) (Figure 3).

3.4. The Expression of HR on Antigen-Specific Plasmablasts. The expressions of various HR on ASC specific to the diarrheal pathogen or the vaccine antigen were studied in five patients with Salmonella diarrhea and in five vaccinees, respectively. In the diarrhea group, the proportion $\alpha_{4} \beta_{7}+$ ASC among all ASC was $94 \pm 6 \%$, L-selectin + ASC $46 \pm 24 \%$, and CLA + ASC $4 \pm 4 \%$. Among vaccinees, the respective figures were $97 \pm 2 \%, 24 \pm 18 \%$, and $1 \pm 2 \%$. These data did not reveal any statistical difference in the HR expressions between diarrhea patients and vaccinees but are consistent with the intestinal homing profile of the migrating cells in both groups.

\section{Discussion}

Investigation of gut-originating plasmablasts from samples of peripheral blood during their recirculation serves as a valuable, less invasive tool for studying intestinal immune response in humans. Instead of examining memory B cells, this approach centers on gut-directed plasmablasts cells spontaneously secreting antibodies against antigens they have encountered recently, that is, the method measures an ongoing intestinal immune response. The transient appearance of these cells in the circulation, which allows only a few days for actual sampling, has posed a severe limitation to this approach. The present study is the first to focus on the kinetics of this response during a natural infection in which the pathogen can persist in the intestine for a longer period of time. It shows that the previously presumed kinetics does 


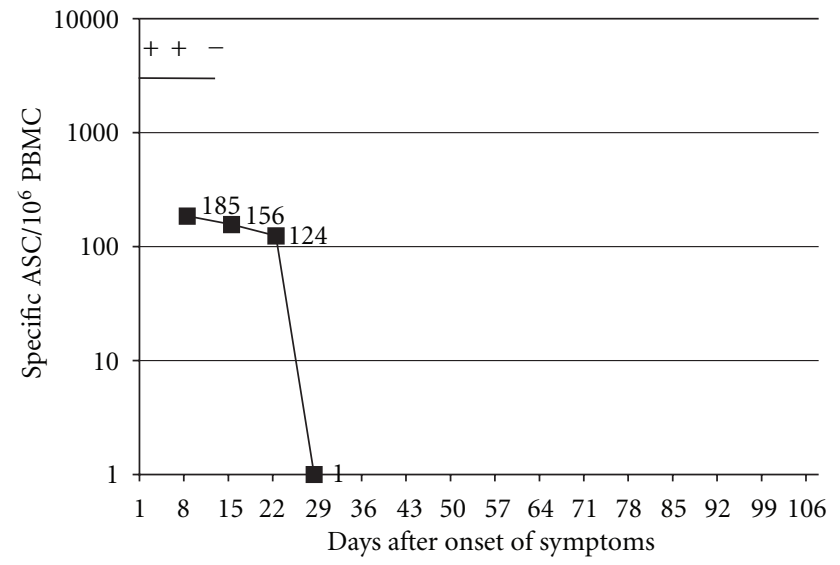

(a)

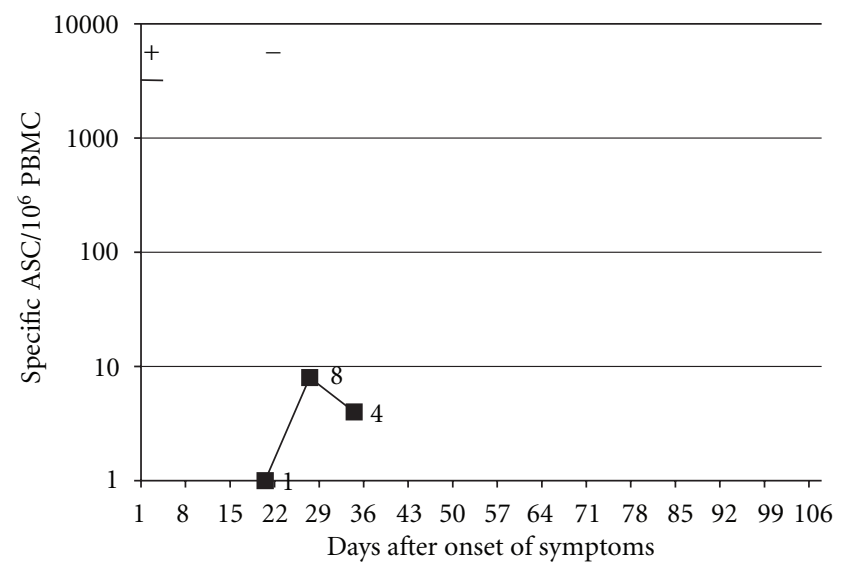

(c)

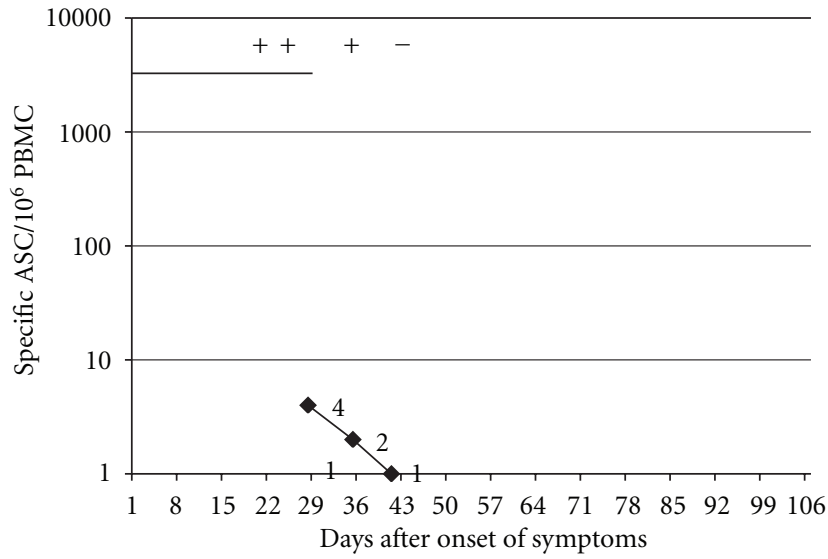

(b)

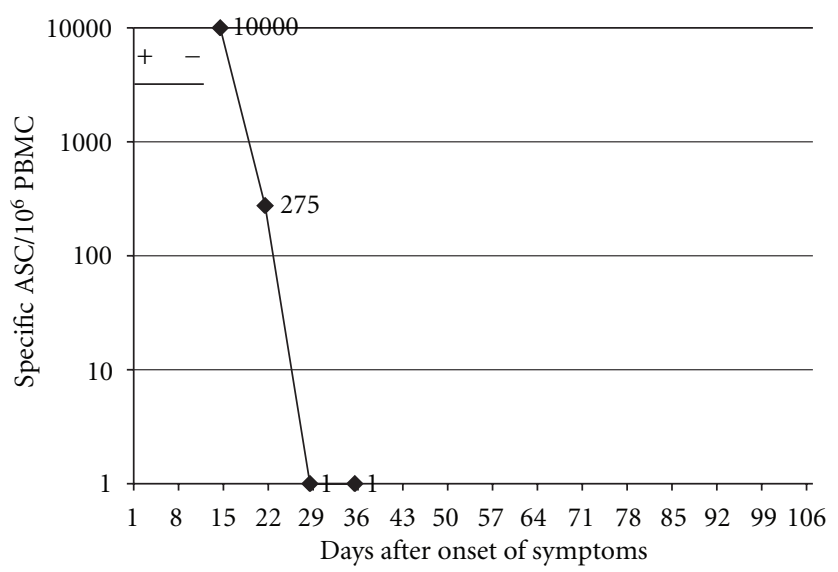

(d)

FIGURE 4: Relation of pathogen-specific circulating plasmablasts (black curve) with symptoms (black horizontal line) and findings in stool samples (+ or -) in four patients with gastroenteritis caused by (a) Salmonella typhimurium, (b) S. emek, (c) S. bradburg, and (d) Campylobacter jejuni. The plots represent each data from one individual. The day of the onset of the symptoms was marked as day 0.

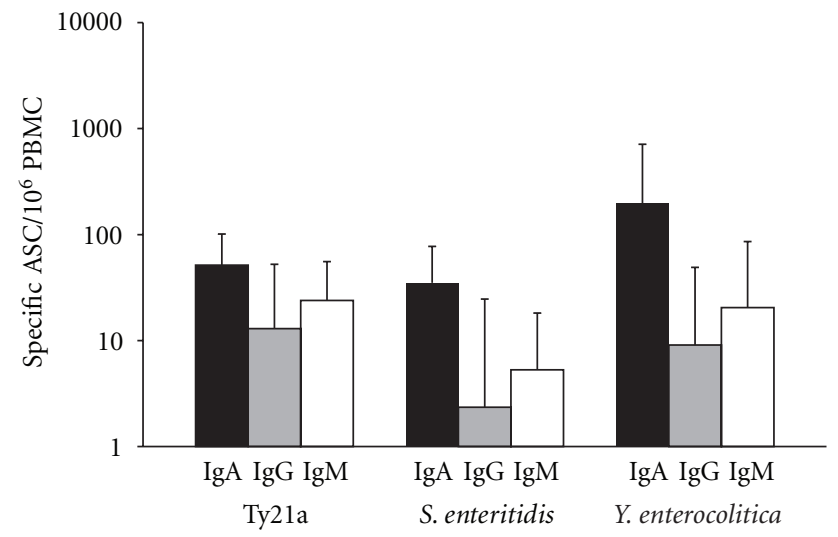

FIGURE 5: The isotype distribution in the peak of the specific plasmablast response in volunteers vaccinated with the oral live Salmonella typhi Ty21a vaccine $(n=6)$ and in patients with gastroenteritis caused by Salmonella enteritidis $(n=8)$ or Yersinia enterocolitica $(n=5)$. The data are given as geometric means of $\mathrm{ASC} / 10^{6} \mathrm{PBMC} \pm \mathrm{SEM}$. not apply to natural infections: if the pathogens persist in the intestine, the actual time frame for sampling can be significantly extended.

4.1. Comparison to Serum Antibody Responses. Assessment of plasmablasts has significant advantages as compared to serum antibody assays. Firstly, plasmablast response measures an immune response to antigens encountered recently, whereas serum antibodies may remain elevated throughout life even if the antigen was encountered decades ago [22]. Secondly, plasmablast assay allows an assessment of the response to each individual's own pathogen. Thirdly, plasmablast response has proved significantly more sensitive than serum antibodies when measuring humoral immune response to intestinal antigen encounter [8]. Our previous studies have shown that shortly after oral vaccination, when antibodies are presumed to exist in intestinal secretions, serum antibodies even fail to rise at all upon intestinal antigen encounter, despite a simultaneous significant plasmablast response [23]. This describes the independent 
nature of systemic and mucosal immune systems and further stresses the role of plasmablast instead of serum antibody measurements when assessing intestinal immune responses: plasmablasts represent intestinal immune response, whereas serum antibodies are mainly produced in the bone marrow. Serum antibodies remaining fairly constant for long periods of time do not provide any information as to whether the pathogen persists in the intestine or the pathogen has been cleared. Up until now, it has been assumed that exploring plasmablasts would not provide such information either, as the cells were thought to be found in the circulation for two weeks only. The continued recruitment of plasmablasts in the present study suggests that the use of the assay of specific plasmablasts can be extended and even applied to the evaluation of antigen persistence in the intestine.

4.2. Significance of the Continuous Recruitment of Plasmablasts. Continuous recruitment of plasmablasts appears to be a means of enhancing the immune response to a pathogen the body has not succeeded in expelling. The generally lower numbers of plasmablasts recruited as the time passes and the even negligible numbers seen occasionally in the later course of the response may be a reflection of antibody responses in the intestine already working against the pathogens. However, such fluctuations in the magnitude of the response with occasional negligible numbers of plasmablasts strongly suggest that a single negative sample does not indicate that the pathogen no longer persists, and therefore, multiple sampling may be required. The highest numbers of plasmablasts are found early in the course of the response. Previous to this we have, in fact, shown that if an oral vaccine is given in six instead of three doses, the plasmablast response is prolonged till day 22 , and even if no clear peak can be seen, the response is at its highest on day 7 [24]. The early peak in the response means that (possible) comparisons in response magnitude between two groups should be carried out on the basis of samples drawn at early stages of the disease, while, in other samples, a qualitative comparison is mainly feasible. It would be interesting to see if chronic carriers of intestinal pathogens (e.g., Salmonella) still have an ongoing recruitment of plasmablasts, as the pathogen can in such cases be regarded to have become part of their regular intestinal microbiota. As to the disappearance of ASC, it is not possible to draw any definite conclusions. In the present data, however, the response seemed to fade away faster after Salmonella (2-3 weeks; Figures 2 and 4 ) than after Yersinia infection (3-7 weeks; Figure 3). As opposed to infections with real multiplying pathogens, the Ty21a vaccine strain is supposed to survive in the intestine only for one or two days. Accordingly, data comparing responses after three versus six doses of Ty21a [24] suggest that in cases with nonreplicating antigens, the plasmablasts disappear from the circulation approximately 8-10 days after the last day of antigen encounter.

Notably, even if the present study focuses on plasmablast response after intestinal antigen encounter, a recirculation of antigen-specific plasmablasts also occurs after nonintestinal encounter [9, 25-27], for example, in infections at other mucosal sites $[10,19,20,28]$ and after parenteral vaccinations $[9,14,25-27,29,30]$. As evidenced by their HR profiles, these plasmablasts are trafficking to nonintestinal sites. Thus, it appears that assaying these cells could be applied to assess persistence of pathogens at nonintestinal sites, too.

\section{Conclusion}

In conclusion, the recruitment of pathogen-specific plasmablasts in the circulation in gastroenteritis has proved less transient than previously reported. Instead, the recruitment seems to continue as the pathogen persists in the intestine, albeit at a lower or, occasionally, even negligible level, indicating that repeated sampling may be necessary. Continued recruitment of plasmablasts in the circulation not only reflects a continuous stimulation of the immune system but also carries potential for assessing immune response to persisting antigens that are suspected to be of significance in the pathogenesis of a disease.

\section{Abbreviations}

ASC: Antibody-secreting cell

CCR: Chemokine receptor

HR: Homing receptor.

\section{Acknowledgments}

The author thanks Professor Heikki Arvilommi for help in planning study and collecting the blood samples. The study was carried out in the Central Hospital of Central Finland and in the Helsinki University Central Hospital.

\section{References}

[1] S. Fagarasan and T. Honjo, "Intestinal IgA synthesis: regulation of front-line body defences," Nature Reviews Immunology, vol. 3, no. 1, pp. 63-72, 2003.

[2] P. Brandtzaeg, "Update on mucosal immunoglobulin A in gastrointestinal disease," Current Opinion in Gastroenterology, vol. 26, no. 6, pp. 554-563, 2010.

[3] H. Sigmundsdottir and E. C. Butcher, "Environmental cues, dendritic cells and the programming of tissue-selective lymphocyte trafficking," Nature Immunology, vol. 9, no. 9, pp. 981-987, 2008.

[4] J. R. Mora and U. H. von Andrian, "Differentiation and homing of IgA-secreting cells," Mucosal Immunology, vol. 1, no. 2, pp. 96-109, 2008.

[5] M. Salmi and S. Jalkanen, "Lymphocyte homing to the gut: attraction, adhesion, and commitment," Immunological Reviews, vol. 206, pp. 100-113, 2005.

[6] E. J. Kunkel and E. C. Butcher, "Plasma-cell homing," Nature Reviews Immunology, vol. 3, no. 10, pp. 822-829, 2003.

[7] A. Kantele, H. Arvilommi, and I. Jokinen, "Specific immunoglobulin-secreting human blood cells after peroral vaccination against Salmonella typhi," Journal of Infectious Diseases, vol. 153, no. 6, pp. 1126-1131, 1986.

[8] A. Kantele, "Antibody-secreting cells in the evaluation of the immunogenicity of an oral vaccine," Vaccine, vol. 8, no. 4, pp. 321-326, 1990. 
[9] A. Kantele, J. M. Kantele, E. Savilahti et al., "Homing potentials of circulating lymphocytes in humans depend on the site of activation: Oral, but not parenteral, typhoid vaccination induces circulating antibody-secreting cells that all bear homing receptors directing them to the gut," Journal of Immunology, vol. 158, no. 2, pp. 574-579, 1997.

[10] M. Quiding-Järbrink, I. Nordström, G. Granström et al., "Differential expression of tissue-specific adhesion molecules on human circulating antibody-forming cells after systemic, enteric, and nasal immunizations. A molecular basis for the compartmentalization of effector B cell responses," Journal of Clinical Investigation, vol. 99, no. 6, pp. 1281-1286, 1997.

[11] S. H. Pakkanen, J. M. Kantele, Z. Moldoveanu et al., "Expression of homing receptors on IgA1 and IgA2 plasmablasts in blood reflects differential distribution of IgA1 and IgA2 in various body fluids," Clinical and Vaccine Immunology, vol. 17, no. 3, pp. 393-401, 2010.

[12] A. Kantele, M. Häkkinen, Z. Moldoveanu et al., "Differences in immune responses induced by oral and rectal immunizations with Salmonella typhi Ty21a: evidence for compartmentalization within the common mucosal immune system in humans," Infection and Immunity, vol. 66, no. 12, pp. 5630-5635, 1998.

[13] A. M. Kantele, R. Takanen, and H. Arvilommi, "Immune response to acute diarrhea seen as circulating antibodysecreting cells," Journal of Infectious Diseases, vol. 158, no. 5, pp. 1011-1016, 1988.

[14] J. M. Kantele, H. Arvilommi, S. Kontiainen et al., "Mucosally activated circulating human B cells in diarrhea express homing receptors directing them back to the gut," Gastroenterology, vol. 110, no. 4, pp. 1061-1067, 1996.

[15] A. M. Gonzalez, M. C. Jaimes, I. Cajiao et al., "Rotavirusspecific B cells induced by recent infection in adults and children predominantly express the intestinal homing receptor $\alpha 4 \beta 7$," Virology, vol. 305, no. 1, pp. 93-105, 2003.

[16] E. P. Bowman, N. A. Kuklin, K. R. Youngman et al., "The intestinal chemokine thymus-expressed chemokine (CCL25) attracts IgA antibody-secreting cells," Journal of Experimental Medicine, vol. 195, no. 2, pp. 269-275, 2002.

[17] C. Berlin, E. L. Berg, M. J. Briskin et al., " $\alpha 4 \beta 7$ integrin mediates lymphocyte binding to the mucosal vascular addressin MAdCAM-1," Cell, vol. 74, no. 1, pp. 185-195, 1993.

[18] A. Kantele, "Peripheral blood antibody-secreting cells in the evaluation of the immune response to an oral vaccine," Journal of Biotechnology, vol. 44, no. 1-3, pp. 217-224, 1996.

[19] A. Kantele, N. Palkola, H. Arvilommi et al., "Local immune response to upper urinary tract infections in children," Clinical and Vaccine Immunology, vol. 15, no. 3, pp. 412-417, 2008.

[20] A. M. Kantele, N. V. Palkola, H. S. Arvilommi, and J. M. Kantele, "Distinctive homing profile of pathogen-specific activated lymphocytes in human urinary tract infection," Clinical Immunology, vol. 128, no. 3, pp. 427-434, 2008.

[21] A. Kantele, J. Zivny, M. Häkkinen, C. O. Elson, and J. Mestecky, "Differential homing commitments of antigen-specific T cells after oral or parenteral immunization in humans," Journal of Immunology, vol. 162, no. 9, pp. 5173-5177, 1999.

[22] I. J. Amanna, N. E. Carlson, and M. K. Slifka, "Duration of humoral immunity to common viral and vaccine antigens," New England Journal of Medicine, vol. 357, no. 19, pp. 19031915, 2007.

[23] A. Kantele and P. H. Makela, "Different profiles of the human immune response to primary and secondary immunization with an oral Salmonella typhi Ty21a vaccine," Vaccine, vol. 9, no. 6, pp. 423-427, 1991.
[24] A. Kantele, "Immune response to prolonged intestinal exposure to antigen," Scandinavian Journal of Immunology, vol. 33, no. 2, pp. 225-229, 1991.

[25] A. Kantele, M. Westerholm, J. M. Kantele, P. H. Mäkelä, and E. Savilahti, "Homing potentials of circulating antibodysecreting cells after administration of oral or parenteral protein or polysaccharide vaccine in humans," Vaccine, vol. 17, no. 3, pp. 229-236, 1999.

[26] C. Lue, A. Tarkowski, and J. Mestecky, "Systemic immunization with pneumococcal polysaccharide vaccine induces a predominant IgA2 response of peripheral blood lymphocytes and increases of both serum and secretory anti-pneumococcal antibodies," Journal of Immunology, vol. 140, no. 11, pp. 37933800, 1988.

[27] R. J. Cox, K. A. Brokstad, M. A. Zuckerman, J. M. Wood, L. R. Haaheim, and J. S. Oxford, "An early humoral immune response in peripheral blood following parenteral inactivated influenza vaccination," Vaccine, vol. 12, no. 11, pp. 993-999, 1994.

[28] F. E. H. Lee, A. R. Falsey, J. L. Halliley, I. Sanz, and E. E. Walsh, "Circulating antibody-secreting cells during acute respiratory syncytial virus infection in adults," Journal of Infectious Diseases, vol. 202, no. 11, pp. 1659-1666, 2010.

[29] T. Nieminen, H. Käyhty, and A. Kantele, "Circulating antibody secreting cells and humoral antibody response after parenteral immunization with a meningococcal polysaccharide vaccine," Scandinavian Journal of Infectious Diseases, vol. 28, no. 1, pp. 53-58, 1996.

[30] T. M. P. T. Herremans, J. H. J. Reimerink, A. M. Buisman, T. G. Kimman, and M. P. G. Koopmans, "Induction of mucosal immunity by inactivated poliovirus vaccine is dependent on previous mucosal contact with live virus," Journal of Immunology, vol. 162, no. 8, pp. 5011-5018, 1999. 


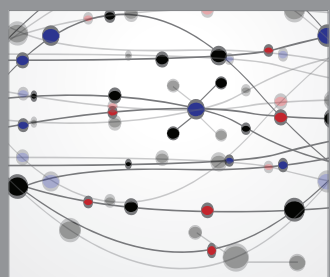

The Scientific World Journal
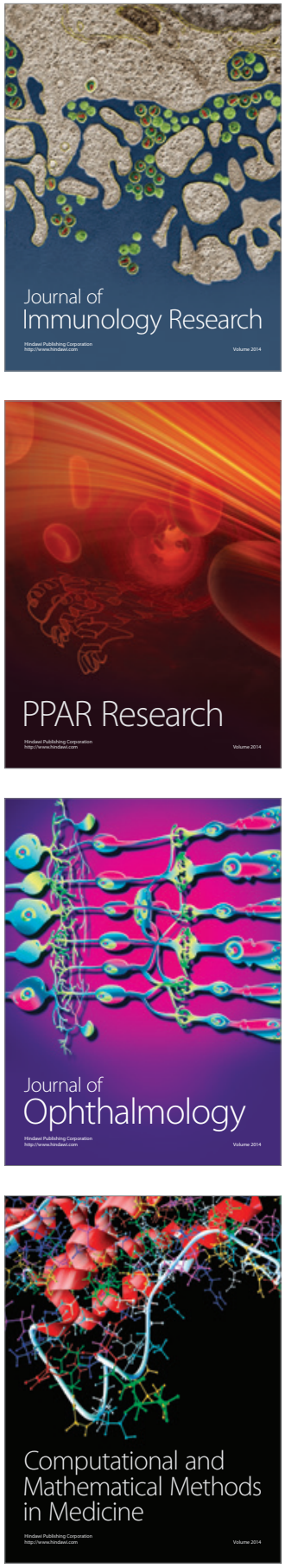

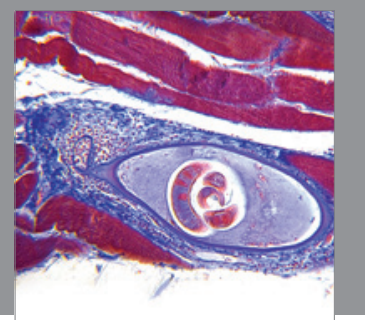

Gastroenterology

Research and Practice
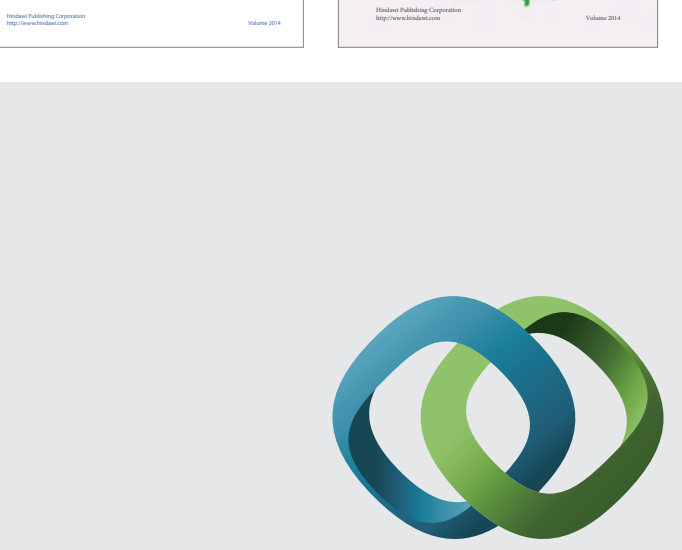

\section{Hindawi}

Submit your manuscripts at

http://www.hindawi.com
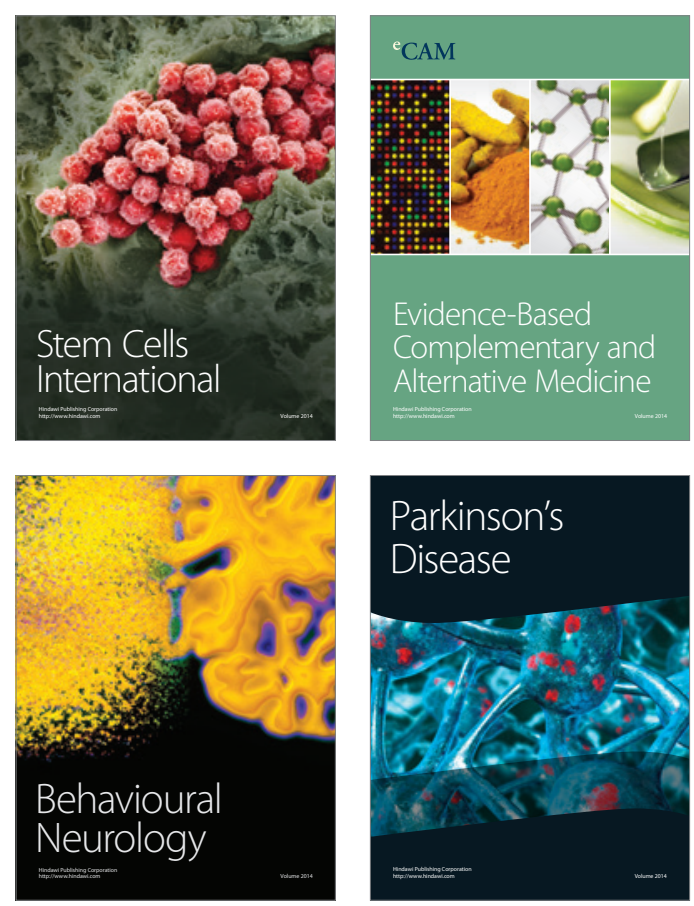

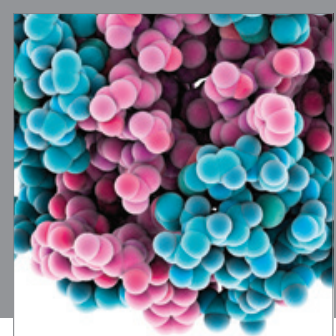

Journal of
Diabetes Research

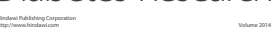

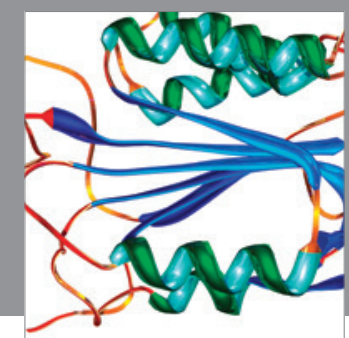

Disease Markers
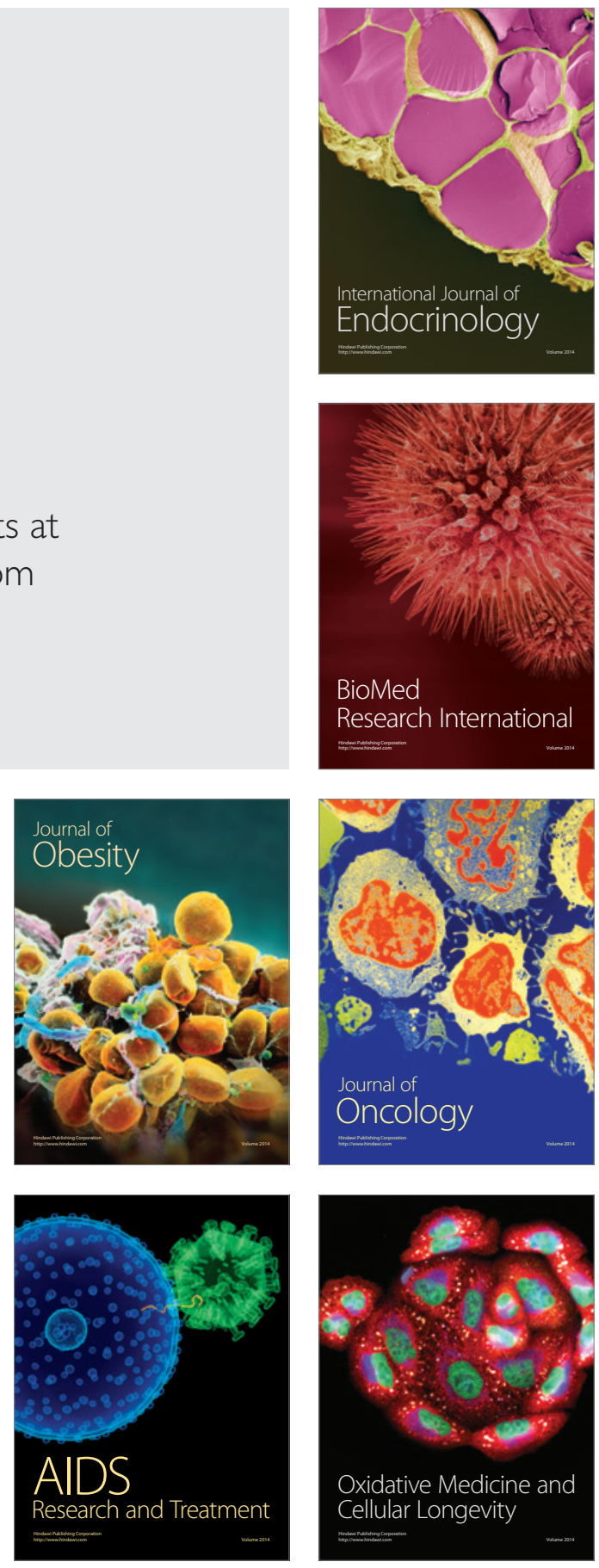